\title{
Radiographic and computed tomographic assessment of the development of the antebrachia and elbow joints in Labrador Retrievers with and without medial coronoid disease
}

\begin{abstract}
Objectives: To compare the development, monitored by radiography and computed tomography, of the antebrachia and elbow joints in seven Labrador Retrievers with healthy elbow joints and in seven Labrador Retrievers that developed medial coronoid disease (MCD), in order to determine whether disturbances in the development of the antebrachia and elbow joints, between the age of six and 17 weeks may lead to medial coronoid disease. Methods: A prospective study of 14 Labrador Retrievers in their active growth stage was performed. The development of the antebrachia and elbow joints was assessed between six and 17 weeks of age using radiography and computed tomography determining the development of secondary ossification centres, radioulnar length ratio, radial angulation, and inter-relationship between the humerus, ulna and radius. Results: For the parameters of ossification of secondary ossification centres, radioulnar length ratio, radial angulation, and joint congruence evaluation, there was no significant difference in the development of the antebrachia and elbow joints of seven Labrador Retrievers positive and seven Labrador Retrievers negative for MCD at the age of six to 17 weeks. Clinical significance: These findings demonstrate that the development of MCD in the Labrador Retrievers in our study was not related to any disturbance in the development of the antebrachia and elbow joints during the rapid growth phase.
\end{abstract}

Keyword: Elbow joint; Labrador Retriever; Medial coronoid disease; Development 